\title{
Psychometric properties of the Persian version of postpartum distress measure scale
}

\author{
Zahra Bakht Shokuhi ${ }^{1}$, Fatemeh Ranjbar ${ }^{1 *} \mathbb{D}$, Sevil Hakimi ${ }^{1,2}$, Rogayeh Bahri $^{3}$ and Saeideh Ghaffarifar ${ }^{4}$
}

\begin{abstract}
Background: The purpose of the present study was to determine psychometric properties of the Persian version of Postpartum Distress Measure Scale (PDM Scale).

Methods: In this psychometric explorative study, the data were collected using a questionnaire containing demographic information, PDM Scale, and Depression and Anxiety Stress Scale-21 (DASS-21). The content, face and construct validity of the questionnaire was examined with participation of ten experts, 10 and 150 women referring to health care centers, who were under common care during their postpartum period, respectively. The concurrent validity of the tool was evaluated using DASS-21. The reliability of the items was evaluated with the participation of 30 women, calculating Cronbach's alpha coefficient and intra-class correlation coefficient.

Results: The Content Validity Index, Content Validity Ratio and Impact Score of the Persian version of the PDM were $0.94,0.73$, and 2.97, respectively. The ten items of the questionnaire were loaded in two factors (general distress and obsessive compulsive symptoms subscale). Those two factors explained $50.78 \%$ of the total variance of women's distress. Internal consistency of the items and stability of the results were confirmed by Cronbach's alpha of 0.72 and Intra-class correlation coefficient of 0.75 .
\end{abstract}

Conclusion: According to the study results, the Persian version of PDM Scale has acceptable psychometric properties. Care providers and researchers can use it as a tool for screening anxiety, depression and obsessivecompulsive disorder in women.

Keywords: Postpartum measure scale, Psychometric properties, Persian

\section{Background}

Postpartum is a very important and stressful period in women and their families" life. During this period, some degrees of worry, anxiety and low mood are normal among women, especially among nulliparous women or the women with unplanned pregnancy. Emotional problems in postpartum period are not limited to depression and can also include symptoms of anxiety and obsessivecompulsive disorders [1].

There are usually some degrees of worry about childbirth, adaptation with postpartum conditions and the birth of a normal baby; however, a percentage of women are more

\footnotetext{
* Correspondence: fk_ranjbar@yahoo.com

${ }^{1}$ Research center of psychiatry and behavioral sciences, Tabriz University of Medical Sciences, Tabriz, Iran

Full list of author information is available at the end of the article
}

worried and experience extreme abnormal fear [2]. In most cases, the main causes of such an abnormal fear are related to the nutritional problems of the baby [3, 4].

On the other hand, physicians who investigate only depression in mothers may be unaware of the distress that women experience [5].

Psychiatric disorders bring undesired consequences for mother and baby, including premature births and low birth weight at birth $[5,6]$. Moreover, children of depressed mothers, who have not been treated during or after pregnancy, are at risk of attachment disorders, poor relationship with the mother and developmental problems [7-9]. For mothers with severe psychiatric disorder, more suicide rates are reported, too [10].

In a study by Glasheen and colleagues (2015), the prevalence of severe psychological distress (SPD) in 
pregnant women was $6.4 \%$ in the past month and in the first trimester and 3.9 in the third trimester. In postpartum women, the rate was $4.6 \%$ in the first two months. It was $6.9 \%$ for $3-5$ months later [10]. In a study by Ahluwalia and associates (2004), the prevalence of SPD was $6.7 \%$, and the prevalence of recent psychological distress; depression; worry and anxiety; and inadequate rest was reported as $12.3,9.9,18.4$, and $34.3 \%$, respectively [11]. In a study by Skari and colleagues (2002), 127 pregnant women and 122 fathers showed that the prevalence of psychological distress in pregnant women and fathers was 37 and 13\%, respectively.

Single parenthood, multi-parity and history of difficult delivery are the risk factors, which contribute to psychological distress [12].

Currently, there are several scales for evaluating patients' anxiety levels. The most important of which are Beck Anxiety Inventory, Spielberger Trait Anxiety Inventory and Edinburgh Postnatal Depression Scale (EPDS), or the 7-item Generalized Anxiety Inventory (GAD-7) [13]. However, clinical manifestations of depression, generalized anxiety disorder, and obsessive-compulsive disorder are not simultaneously screened by these questionnaires. In practice, such a problem leads to less desired diagnosis of postpartum mental distress, lower rate of referrals and ineffective therapeutic approaches. In this regard, a study by Goodman and collegues (2010) found that $17 \%$ of the patients had symptoms of depression or anxiety disorders 6 weeks after delivery; however, only one quarter of the patients were screened; two percentage of them were referred and received adequate treatment [14].

Allison and associates (2011) developed and validated postpartum distress measure scale. This scale measures the symptoms of postpartum distress. This is the first postpartum screening tool, which is specific for simultaneous screening of anxiety and depression symptoms. With this scale, symptoms of generalized distress and obsessive-compulsive disorder are assessed reliably. The use of this Post-partum Distress Measure (PDM) Scale increases the possibility of diagnosing any significant clinical symptoms, including clinical history of depression, generalized anxiety disorder, and obsessivecompulsive disorder, which is reported in two-thirds of postpartum women [15].

Up to the time of this research and to the best of our knowledge and literature review, there was no Persian scale for evaluating postpartum distress. Therefore, the present study was aimed to translate and adapt the original version of PDM Scale and determine the psychometric properties of its Persian version.

\section{Methods}

This psychometric explorative study was conducted in health centers, affiliated to Tabriz University of Medical
Sciences, from April 2015 to March 2016. The data collection tool was the English version of the Postpartum Distress Measure Scale (PDM Scale). The ethics approval was obtained from university review board (No. IR.TBZMED.REC 1395.660). Permission for translation and adaptation of the original questionnaire into Persian language was obtained from the authors of the English version of PDM Scale through an e-mail.

Translation and adaptation were done using ForwardBackward translation approach. For this purpose, one psychiatrist and one reproductive health specialist separately translated the original version of the questionnaire into Persian. The two translated versions were compared and the differences and ambiguities were identified and resolved. Then, the final Persian version was translated into English by one bilingual translator who did not participate already in the study. Finally, in a committee by the presence of the translators and the research team, the English version of the questionnaire was compared with the original version and all semantic problems were resolved.

\section{Statistical analysis Assessment of the content validity}

After the stages of translation and preparation of the Persian version of the questionnaire, the content and face validity of the questionnaire were evaluated in both qualitative and quantitative ways. For so doing, ten experts, including eight psychiatrists, one reproductive health specialist and one psychologist, provided written comments on how to observe the grammar, the use of proper words, the placement of the items in their proper place and appropriate scoring of the items. In order to assess content validity, Content Validity Ratio (CVR) and Content Validity Index (CVI) were calculated.

In order to determine"CVR, the experts were asked to examine each item based on 3-part Likert scale (it is necessary; useful, but not necessary; not necessary).

To calculate CVI, relevance, simplicity and clarity of the items were examined, using a four-part Likert scale (for instance: $1=$ not relevant, $2=$ relatively relevant, $3=$ relevant and $4=$ completely relevant).

Using the formula of $V R=\frac{\mathrm{Ne}-\mathrm{N} / 2}{\mathrm{~N} / 2}$, CVR was calculated. Lawseh table was used to decide the values. Since 10 specialists completed the questionnaires, any score more than 0.62 was considered to be acceptable for confirming the content validity of each item.

CVI was calculated by the formula of C VI $=\frac{\text { The number of specialists who gave } 3 \text { and } 4 \text { for the items }}{\mathrm{N}}$ and CVIs over 0.79 were considered acceptable.

\section{Assessment of the face validity}

Face validity of the questionnaire was evaluated by a panel of experts, including eight psychiatrists, one 
reproductive health specialist and one psychologist and ten women with postpartum distress, in two qualitative and quantitative methods. For qualitative evaluation, face-to-face interviews were conducted with ten participating women. Difficulty in understanding the words and the possibility of any misunderstandings and ambiguity in the meaning of the phrases were investigated. Those ten participating women were randomly selected from 5 health centers (two women from each center). For quantitative evaluation, the experts evaluated the degree of appropriateness of each item, based on a 5-point Likert scale (very important $=5$, somehow important $=4$, moderately important $=3$, slightly important $=2$, and not important $=1$ ). In doing so, the impact score of each item was calculated. Impact scores (IS) were calculated by the following formula: Impact Score $=$ Frequency (\%) $\times$ Importance. Impact score of each item had to be above 1.5 and the items with IS over 1.5 were considered acceptable in terms of face validity.

\section{Assessment of the construct validity}

Construct validity of the questionnaire was studied by exploratory factor analysis. Criterion-related validity (concurrent validity) was evaluated through correlation test between the results of two questionnaires of DASS21 and PDM Scale. Factor analysis was performed to reduce the data, explain and clarify the theoretical structure of the questionnaire. Sampling adequacy was studied by KMO (Kaiser-Meyer-Olkin) Measure and Bartlett's test of Sphericity. The data were extracted by principal component method and the rotation of the factors by Varimax method.

In order to assess the construct validity of the questionnaire, random and multi-stage sampling was done. After the random selection of 15 centers from the list of all health centers in Tabriz, the cases of women who were in postpartum period were examined and from each center, a number of cases were randomly selected based on the total number of available cases.

In this phase, 150 women with postpartum distress were recruited. As the women with postpartum distress have similar clinical manifestations all around the country, the women in Tabriz were considered to be representative of other women in Iran. In Iran, governmental facilities are provided for all women, who have just given birth to their children and they are regularly visited at health centers after delivery. As those facilities are provided for free, almost all women refer to health centers. So, access to the women with postpartum distress is highly provided at those health centers. In other words, employing participants from health centers increased the feasibility of our study.

Women who announced their written informed consent participated in the study. The questionnaire was completed for all referring women who had symptoms or signs of distress. Women with a probable diagnosis were visited by a psychiatrist and those who were approved to suffer from any degrees of postpartum distress were recruited in the present study.

The inclusion criteria were: aged $19-40$ years and being referred during the first year after delivery. Exclusion criteria included: having a diagnosed psychiatric disease or history of high risk pregnancy, including gestational diabetes, pre-eclampsia, fetal growth retardation, twin pregnancy, instrumental delivery and fetal abnormalities.

Data collection tools in this study included three questionnaires. All three questionnaires were completed for all participants. In general, for those who did not have reading and writing literacy, questions were read by the main researcher and the responses given by the participants were re-checked and marked by her.

The first questionnaire included baseline characteristics of the participants: including their age, marital status, job, education level and their family economic status.

The second questionnaire was the Persian version of PDM scale. Both the face and content validity of this questionnaire is evaluated in the present study. This questionnaire has 10 items. The completion time of each questionnaire was five minutes. The participating women, by assigning a score in the range of 0 to 3, evaluated the severity of each of the symptoms they felt. A four-point Likert Scale (not at all = 0, low =1, medium = 2 , and high $=3$ ) was used for scoring each item. Except for the items number one, six and ten, other items were reversely scored. The minimum and maximum scores are zero and 48, respectively. The higher the score, the greater the distress [15].

\section{Concurrent validity assessment}

DASS-21 was the third questionnaire, which was used in this study. It was used to determine the concurrent validity of the Persian version of Postpartum Distress Measure. This scale provides valid and reliable assessment of the three constructs of depression, anxiety and stress [16]. The internal consistency and validity of DASS-21 is within an acceptable to excellent range. According to some studies, its 21 -item version has several advantages over its full 42 -item version, so its use is preferred to the use of its full version [15]. The main DASS-21 questionnaire consists of three subscales of depression, anxiety and stress, and a total of 21 items. Each sub-scale consists of seven items. Its items address the status of patients' symptoms in the last week. Each item has a score of 0 to 3 (very true to $m e=3$, it is pretty true to $m e=2$, to some extent true to $m e=1$, and by no means true to $m e=0$ ). For each sub-scale, the range of scores can be from 0 to 21 . In the 21-question version, the sub-scales 
are multiplied by two. The higher the scores, the higher the severity of depression, anxiety and stress [15]. That questionnaire was translated into Persian by Asghari and colleagues in 2008. Its Persian version has appropriate psychometric properties. The internal consistency coefficients of depression, anxiety and stress scales were 0.93 , 0.90 , and 0.92 , respectively. Intra-class correlation coefficients of depression, anxiety and stress subscales were $0.84,0.89$, and 0.90 , respectively [17].

\section{Reliability assessment}

The internal consistency of the items was studied by calculating Cronbach's alpha coefficient. Stability of the results was examined applying test-retest method and calculating intra-class correlation coefficient (ICC). For this purpose, the questionnaire was completed twice by 30 women with postpartum distress during a 10 day interval.

\section{Quality supervision of the study}

In order to implement the quality supervision of the study, the research questionnaire was administered to the meticulous and caring participants, whether the experts or the women with postpartum distress. Content Validity Index (CVI) and Content Validity Ratio (CVR) were determined by two different groups of experts. In addition, the data of the completed questionnaires were manually checked for any careless completing or responding before the data were analyzed.

As all questionnaires were completed in the presence of health care providers, there was no missing data in this study; however, in case of any missing data, it was planned to perform sensitivity analyses, missing data patterns analysis or imputation and of course reporting.

Statistical analysis was performed using SPSS software, version 18 (IBM, Armonk, NY, USA).

\section{Results}

In this study, 150 mothers participated in their postpartum period. The mean age of the participants was $29.92 \pm 5.45$ years. $85.3 \%$ of the participants were households, $75.3 \%$ of the women had no history of abortion, and $53.3 \%$ of the new-borns were girls. $98 \%$ of women were satisfied with their child gender and $87.3 \%$ of mothers were satisfied with their pregnancy. Spouse's support for participants was high and moderate among 77.3 and $17.3 \%$ of the women, respectively. Other baseline characteristics of the participating women are presented in Table 1.

The Content Validity Index, Content Validity Ratio and Impact Score of the Persian version of the PDM were $0.94,0.73$, and 2.97 , respectively.

The results for the content and face validity of the items are presented in Table 2. The communalities of 10
Table 1 Baseline characteristics of the women participating in the psychometric study of the Persian version of Postpartum Distress Measure scale $(n=150)$

\begin{tabular}{lll}
\hline Variable & & \\
\hline & & Mean \pm SD (Min to Max) \\
Age & & $29.92 \pm 5.45$ (19 to40) \\
Spouse age & & $34.51 \pm 5.75$ (22 to 55) \\
& & Frequency (percentage) \\
Education level & Illiterate & $22(14.6)$ \\
& High school & $58(38.7)$ \\
& University & $70(46.7)$ \\
Spouse job & Unemployed & $1(0.7)$ \\
& Employed & $29(19.3)$ \\
& Worker & $34(22.7)$ \\
& Self-employed & $52(34.6)$ \\
& Driver & $24(16)$ \\
& Others & $10(6.7)$ \\
Spouse education Level & Illiterate & $1(0.7)$ \\
& High school & $63(42)$ \\
& University & $86(57.3)$
\end{tabular}

items of PDM Scale are shown in Table 3. Ten items with eigenvalues greater than one were extracted. These 10 items in two factors were able to explain $50.78 \%$ of the total variance. All items of the questionnaire were loaded in two factors. Factor load scree plot is shown on Fig. 1.The naming of each of the factors was agreed based on the original version of the questionnaire.

The initial and rotated eigenvalues of two-factor PDM Scale are shown in Table 4.

According to the results of exploratory factor analysis, two primary factors were extracted. The first factor called "General Distress", explained 37.24\% of the variance. The second factor called "obsessive-compulsive" explained $13.53 \%$ of the variance. The factor load for the items is shown in Table 5. Considering the importance of suicidal thoughts (item number seven), the research team decided not to eliminate item seven.

At the stage of determining criterion-related (concurrent) validity, the correlation between general distress and anxiety, depression and stress was $0.531,0.422$ and 0.460 , respectively $(P=0.001)$. The correlation coefficient between obsessive compulsive and anxiety, depression or stress was $0.412,0.396$ and $0.562(P=0.001)$.

Intra-class correlation coefficient for the construct of general distress was 0.68 .It was 0.73 for the construct of obsessive compulsive and 0.75 for the whole questionnaire $(P=0.001)$.

In order to investigate the homogeneity of constructs, Cronbach's alpha coefficient was calculated. Cronbach's alpha for the factor of general distress was 0.60. It was 
Table 2 The results for the content and face validity of the Persian version of Postpartum Distress Measure Scale

\begin{tabular}{|c|c|c|c|c|c|}
\hline \multirow[t]{2}{*}{ Item } & \multicolumn{3}{|c|}{ Content Validity Index } & \multirow{2}{*}{$\begin{array}{l}\text { Content Validity } \\
\text { Ratio }\end{array}$} & \multirow{2}{*}{$\begin{array}{l}\text { Impact } \\
\text { Score }\end{array}$} \\
\hline & Relevance & Clarity & Simplicity & & \\
\hline I feel sad and hopeless & 0.8 & 0.9 & 1 & 0.8 & 3.8 \\
\hline I am crying more than usual & 0.9 & 0.9 & 0.8 & 0.8 & 1.5 \\
\hline I cannot make decisions or concentrate & 0.9 & 1 & 1 & 0.6 & 1.7 \\
\hline I feel overwhelmed & 0.9 & 1 & 1 & 0.8 & 4.7 \\
\hline I'm afraid I will never feel better & 1 & 1 & 0.8 & 0.7 & 3.7 \\
\hline I think about taking my own life & 0.9 & 0.9 & 0.8 & 0.7 & 2.5 \\
\hline I have recurring thoughts about harm coming to my baby, my family, or myself & 1 & 1 & 1 & 0.7 & 3.2 \\
\hline I have recurring thoughts about my baby getting sick or having some kind of problem & 1 & 1 & 1 & 0.8 & 2.5 \\
\hline I check on my baby multiple times throughout the night & 1 & 0.9 & 0.8 & 0.7 & 3.4 \\
\hline I have thoughts about my baby that scare me & 1 & 1 & 1 & 0.7 & 2.7 \\
\hline The mean of the measures for the whole questionnaire & 0.94 & 0.96 & 0.92 & 0.73 & 2.97 \\
\hline
\end{tabular}

0.62 for the factor of obsessive compulsive and 0.72 for the PDM scale in all.

Sampling adequacy was confirmed by KMO of 0.811 and significance of 0.000 of the Bartlett's test of Sphericity.

\section{Discussion}

The present study aimed to determine the psychometric properties of the Persian version of PDM scale. Until the writing of the present paper, few studies have examined psychometric properties of the PDM scale in other languages $[15,18]$.

Exploratory factor analysis method was used to assess the construct validity of the Persian version of the questionnaire. In doing so, with respect to the eigenvalues higher than one and scree plot, two factors were extracted. This two-factor questionnaire with 10 questions can be easily and quickly practiced. Typically, in factor analysis, the percentage of communalities less than 0.5 is not acceptable, and the scores of such items should be eliminated; however, considering the importance of suicidal thoughts (item number seven), the research team decided not to eliminate item seven. The decision for maintaining such an important item is confirmed by statistical considerations in data analysis [19]. These results were similar to the results of factor analysis of the original questionnaire [15]. In a study by Hirsch et al. (2017), six questions were related to general depression and four questions were about obsessive-compulsive symptoms, while the question of suicide was not present among the factors [18]. The results of both studies were consistent with the present study. These results suggest that, despite the cultural differences in psychological variables in different communities, concerns about issues and problems related to pregnancy are universal.

Cronbakh's Alpha for the PDM in this study was 0.75 and its ICC was $0.75(\mathrm{CI}=0.7$ to 0.82 ).

These numbers confirm that PDM Scale is a reliable and coherent and applicable tool in the clinical environment. In the original study, Cronbach's alpha for the English version of the PDM Scale, general distress and obsessive compulsive symptoms sub-scales was 0.88 , 0.91 and 0.83 , respectively [15], indicating a desirable reliability. Also, in another study by Hirsch et al. (2017),

Table 3 Communalities of the 10 items of the Persian version of Postpartum Distress Measure Scale

\begin{tabular}{ll}
\hline Item & Communality \\
\hline I feel sad and hopeless & 0.537 \\
I am crying more than usual & 0.460 \\
I cannot make decisions or concentrate & 0.570 \\
I feel overwhelmed & 0.455 \\
I'm afraid I will never feel better & 0.617 \\
I think about taking my own life & 0.758 \\
I have recurring thoughts about harm coming to my baby, my family, or myself & 0.223 \\
I have recurring thoughts about my baby getting sick or having some kind of problem & 0.433 \\
I check on my baby multiple times throughout the night & 0.476 \\
\hline
\end{tabular}




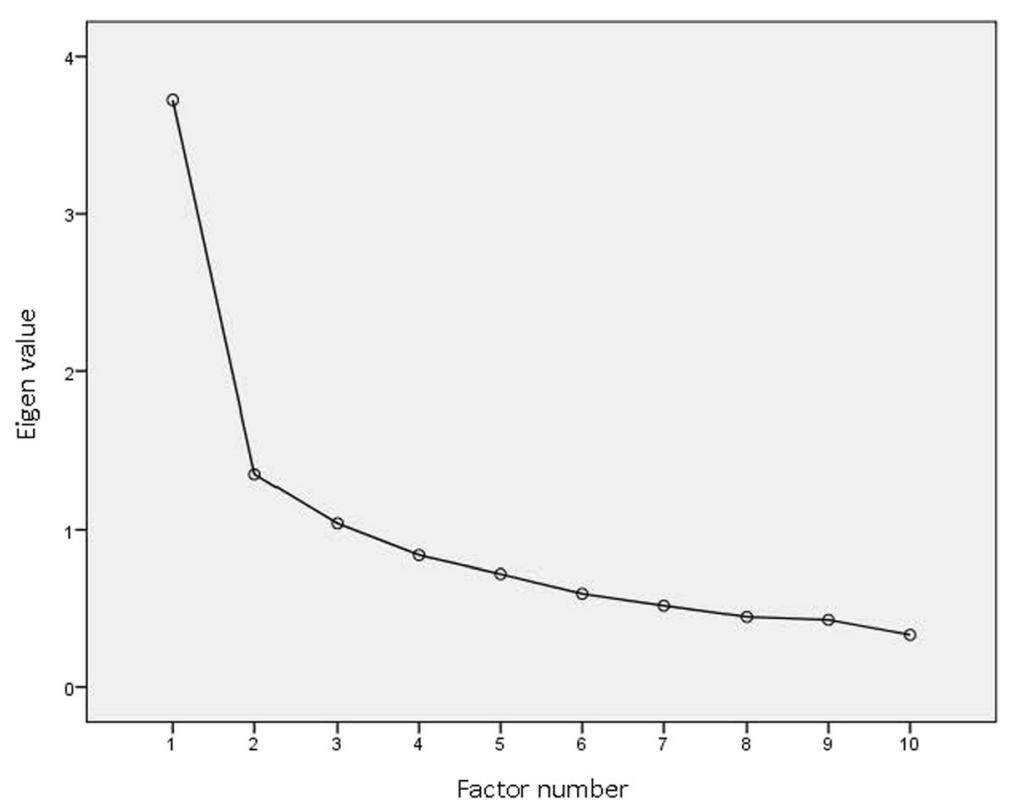

Fig. 1 Factor load scree plot of the items for determining the number of extracted factors of the Persian version of Postpartum Distress Measure Scale

Cronbach's alpha for the whole questionnaire, the general distress and the obsessive-compulsive subscales was 0.85 , 0.84 and 0.80, respectively [18]. Although Cronbach Alpha correlation coefficient depends on the number of questions, the high Cronbach alpha does not show that tool is one dimensional; therefore, the low internal stability of the tool may be related to the low number of its questions [20]. On the other hand, the reliability of a questionnaire is indicative of the degree of measurement stability and proportionality of the items with each other [21]. According to the findings of this study, the psychometric properties of the Persian version of the PDM scale is desirable [22].

Study of criterion validity of the questionnaire showed that the results from the Persian version of PDM are highly correlated with the results from Cambridge pregnancy Distress Measure, which is as a valid and reliable tool. It seems that the Persian version of Postpartum Distress Measure evaluates constructs similar to the Cambridge pregnancy Distress Measure and it can be used as an acceptable tool for screening mental problems during postpartum period [23].

In the present study, the value of KMO was 0.811 . $\mathrm{KMO}$ measure is an index for the sample size adequacy.
Values greater than 0.6 are acceptable and the values closer to one are better [24]. Bartlett's Sphericity test was used to determine the fitting of the model, which had a significant level. In Bartlett Sphericity test, if the significance value is less than 0.5 , the ability to validate the data is confirmed. The two criteria mentioned are important indicators for confirming the adequacy of sampling and the ability to factor analysis of the items.

\section{Limitations of the study}

Since the psychometric properties of the PDM scale in languages other than Persian was not already investigated, the researchers compared the psychometric properties of the Persian version of the questionnaire with only those of its original English version.

Moreover, in some women, distress during pregnancy imay be due to causes other than pregnancy. The main designers of PDM Scale have stated that the tool measures only the specific pregnancy outcomes; however, since our patients have not been evaluated and screened for physical or psychological problems, it is recommended to evaluate other psychological problems of the

Table 4 Initial and rotated eigenvalues of 2-factors of the Persian version of Postpartum Distress Measure Scale

\begin{tabular}{|c|c|c|c|c|c|c|}
\hline \multirow[t]{2}{*}{ Factor } & \multicolumn{3}{|c|}{ Initial eigenvalue } & \multicolumn{3}{|c|}{ Total rotated square } \\
\hline & Total & Variance $\%$ & Cumulative $\%$ & Total & Variance $\%$ & Cumulative $\%$ \\
\hline 1 & 3.72 & 37.24 & 37.24 & 2.95 & 29.59 & 29.59 \\
\hline 2 & 1.35 & 13.53 & 50.78 & 2.11 & 21.19 & 50.78 \\
\hline
\end{tabular}


Table 5 Factor load of items of the Persian version of Postpartum Distress Measure Scale

\begin{tabular}{|c|c|c|c|}
\hline \multirow[t]{2}{*}{ Item } & \multicolumn{3}{|c|}{ Factor load } \\
\hline & 1 & 2 & 3 \\
\hline I feel sad and hopeless & 0.892 & 0.100 & - \\
\hline I am crying more than usual & 0.649 & - & - \\
\hline I cannot make decisions or concentrate & 0.727 & - & - \\
\hline I feel overwhelmed & 0.440 & 0.480 & - \\
\hline I'm afraid I will never feel better & 0.784 & - & - \\
\hline I think about taking my own life & 0.532 & 0.680 & - \\
\hline I have recurring thoughts about harm coming to my baby, my family, or myself & 0.395 & 0.448 & - \\
\hline I have recurring thoughts about my baby getting sick or having some kind of problem & 0.281 & 0.660 & 0.425 \\
\hline I check on my baby multiple times throughout the night & 0.395 & 0.590 & - \\
\hline I have thoughts about my baby that scare me & - & 0.339 & - \\
\hline
\end{tabular}

women in future studies, in order to yield more reliable results.

\section{Recommendations}

"A cut off score is determined when a scale is criterionreferenced. In criterion-referenced scales, scores below the cut off represent the absence of the intended attribute. PDM scale is a norm-referenced scale and lower scores represent lower distress, not its absence. Hence, there was no need to determine the cut off score for this screening tool; however, if it is intended to apply it as a sensitive diagnostic tool, it is recommended to employ appropriate methods to determine its optimal cut off score", so that the Persian version can be easily used for diagnosing clinical cases.

\section{Conclusion}

According to the study results, the Persian version of PDM Scale has a coherent structure and acceptable psychometric properties. Applying this valid and reliable questionnaire, psychiatrists, other care providers and researchers can separately or simultaneously screen anxiety, depression and obsessive-compulsive disorder in women. It means that the PDM can be a helpful tool in identifying a broader range of postpartum distress, including obsessive-compulsive symptoms that were formerly neglected in clinical screening measures.

\section{Abbreviations}

CVI: Content Validity Index; CVR: Content Validity Ratio; DASS-21: Depression and Anxiety Stress Scale-21; EPDS: Edinburgh Postnatal Depression Scale; GAD7: Generalized Anxiety Inventory; ICC: Intra-class Correlation Coefficient;

IS: Impact score; KMO: Kaiser-Meyer-Olkin; PDM Scale: Postpartum Distress Measure Scale; SPD: Severe Psychological Distress; SPSS: Statiscal Package for social science

\section{Acknowledgements}

This research was conducted as a part of a thesis of PGY4 at psychiatry department at Tabriz University of Medical Sciences. Hereby, assistance, cooperation and funding of research deputy of Tabriz University of Medical Science and the invaluable participation of women would be appreciated.

\section{Authors' contributions}

All authors approved the study. ZB conceived and the study; collected and analyzed the data; interpreted the findings; wrote the first draft of the manuscript; read and critically revised the first draft and confirmed the final version of the manuscript. FR conceived and the study; analyzed the data; interpreted the findings; contributed to the validation study of the research questionnaire; read and critically revised the first draft and confirmed the final version of the manuscript. SH analyzed the data; interpreted the findings; contributed to the validation study of the research questionnaire; read and critically revised the first draft and confirmed the final version of the manuscript. RB collected and analyzed the data; interpreted the findings; contributed to the validation study of the research questionnaire; read and critically revised the first draft and confirmed the final version of the manuscript. SGH analyzed the data; interpreted the findings; contributed to the validation study of the research questionnaire; read and critically revised the first draft and confirmed the final version of the manuscript.

\section{Funding}

This study is a part of a Psychiatry specialty thesis of the main researcher (ZB). Conducting this study is supported by Tabriz University of Medical Sciences. The funding body had no role in the design of the study and collection, analysis, and interpretation of data and in writing the manuscript. We have not received external funding from a commercial organization.

\section{Availability of data and materials}

The data sets generated and/or analyzed during the current study will not publicly available before publication of its pertinent manuscripts, but are available from the corresponding author on reasonable request.

\section{Ethics approval and consent to participate}

Conducting this study was approved by the ethical committee board at Tabriz University of Medical Sciences. The approval code is IR.TBZMED.REC 1395.660 .

All women voluntarily participated in this study and they completed the research questionnaires with their consent. The consent obtained from participants was written. The learning objectives of the study were clearly shared with experts and participating women. A unique code was assigned to each woman. The participants were told that all the research data will be saved confidential. They were granted the right to withdraw from the study whenever they wanted.

\section{Consent for publication}

Not applicable.

\section{Competing interests}

The authors declare that they have no competing interests.

\section{Author details}

${ }^{1}$ Research center of psychiatry and behavioral sciences, Tabriz University of Medical Sciences, Tabriz, Iran. ${ }^{2}$ School of Nursing and Midwifery, Tabriz 
University of Medical Sciences, Tabriz, Iran. ${ }^{3}$ Department of clinical psychology, Fabric branch, Islamic Azad University, Tabriz, Iran. ${ }^{4}$ Medical Education Research Centre, Health Management and Safety Promotion Research Institute, Tabriz University of Medical Sciences, Tabriz, Iran.

Received: 18 May 2019 Accepted: 14 February 2020

Published online: 27 February 2020

\section{References}

1. Health NCCFM, editor Antenatal and Postnatal Mental Health: Clinical Management and Service Guidance: Updated edition2014: British Psychological Society.

2. Mistry R, Stevens GD, Sareen H, De Vogli R, Halfon N. Parenting-related stressors and self-reported mental health of mothers with young children. Am J Public Health. 2007:97(7):1261-8.

3. Hung $\mathrm{CH}$. Revalidation of the postpartum stress scale. J Clin Nurs 2006;15(6): 718-725. Epub 2006/05/11. doi: https://doi.org/10.1111/j.1365-2702.2006. 01350.x. PubMed PMID: 16684167.

4. Fredriksson GE, Hogberg U, Lundman BM. Postpartum care should provide alternatives to meet parents' need for safety, active participation, and 'bonding'. Midwifery. 2003;19(4):267-276. Epub 2003/11/19. PubMed PMID: 14623506

5. Winter L, Colditz PB, Sanders MR, Boyd RN, Pritchard M, Gray PH, et al. Depression, posttraumatic stress and relationship distress in parents of very preterm infants. Arch Womens Ment Health 2018;21(4):445-451. Epub 2018/ 03/05. doi: https://doi.org/10.1007/s00737-018-0821-6. PubMed PMID: 29502280 .

6. Sabuncuoglu O, Basgul A. Pregnancy health problems and low birth weight associated with maternal insecure attachment style. J Health Psychol 2016; 21(6):934-943. Epub 2014/07/30. doi: https://doi.org/10.1177/ 1359105314542819. PubMed PMID: 25070968

7. Matsunaga A, Takauma F, Tada K, Kitamura T. Discrete category of motherto-infant bonding disorder and its identification by the mother-to-infant bonding scale: a study in Japanese mothers of a 1-month-old. Early Hum Dev 2017;111:1-5. Epub 2017/05/20. doi: https://doi.org/10.1016/j. earlhumdev.2017.04.008. PubMed PMID: 28525876.

8. Ohashi Y, Sakanashi K, Tanaka T, Kitamura T. Mother-to-infant bonding disorder, but not depression, 5 days after delivery is a risk factor for neonate emotional abuse: A study in Japanese mothers of 1-month olds. Open Fam Stud J. 2016;8(1)

9. Neri E, Agostini F, Baldoni F, Facondini E, Biasini A, Monti F. Preterm infant development, maternal distress and sensitivity: The influence of severity of birth weight. Early human development. 2017;106-107:19-24. Epub 2017/ 02/13. doi: https://doi.org/10.1016/j.earlhumdev.2017.01.011. PubMed PMID: 28189798

10. Glasheen C, Colpe L, Hoffman V, Warren LK. Prevalence of serious psychological distress and mental health treatment in a national sample of pregnant and postpartum women. Matern Child Health J 2015;19(1):204216. Epub 2014/05/08. doi: https://doi.org/10.1007/s10995-014-1511-2. PubMed PMID: 24802262

11. Ahluwalia IB, Mack KA, Mokdad A. Mental and physical distress and high-risk behaviors among reproductive-age women. Obstet Gynecol. 2004;104(3): 477-83.

12. Skari H, Skreden M, Malt UF, Dalholt M, Ostensen AB, Egeland T, et al. Comparative levels of psychological distress, stress symptoms, depression and anxiety after childbirth - a prospective population-based study of mothers and fathers. BJOG : an international journal of obstetrics and gynaecology. 2002;109(10):1154-63.

13. Cox JL, Chapman G, Murray D, Jones P. Validation of the Edinburgh postnatal depression scale (EPDS) in non-postnatal women. J Affect Disord 1996;39(3):185-189. Epub 1996/07/29. PubMed PMID: 8856422.

14. Goodman JH, Tyer-Viola L. Detection, treatment, and referral of perinatal depression and anxiety by obstetrical providers. Journal of women's health (2002). 2010;19(3):477-90. Epub 2010/02/17. doi: https://doi.org/10.1089/jwh. 2008.1352. PubMed PMID: 20156110.

15. Allison KC, Wenzel A, Kleiman K, Sarwer DB. Development of a brief measure of postpartum distress. J Women's Health 2011;20(4):617-623. Epub 2011/03/19. doi: https://doi.org/10.1089/jwh.2010.1989. PubMed PMID: 21413895.

16. Le MTH, Tran TD, Holton S, Nguyen HT, Wolfe R, Fisher J. Reliability, convergent validity and factor structure of the DASS-21 in a sample of
Vietnamese adolescents. PloS one. 2017;12(7):e0180557. Epub 2017/07/21. doi: https://doi.org/10.1371/journal.pone.0180557. PubMed PMID: 28723909; PubMed Central PMCID: PMCPMC5516980.

17. Asghari A, Saed F, Dibajnia P. Psychometric properties of the depression anxiety stress Scales-21 (DASS-21) in a non-clinical Iranian sample. Int J Psychol. 2008;2(2):82-102.

18. Hirsch NM, Fingerhut R, Allison KC. The Prenatal Distress Measure: Adaptation of the Postpartum Distress Measure for a Prenatal Sample. Journal of women's health (2002). 2017;26(11):1193-200. Epub 2017/04/14. doi: https://doi.org/10.1089/jwh.2016.5962. PubMed PMID: 28402697.

19. Schabenberger O, Gotway CA. Statistical methods for spatial data analysis: CRC press; 2017

20. Garrett LE, Klemme AK, Wilkins AL. 7. Related constructs and measures from beyond the field of ethics. Research Companion to Ethical Behavior in Organizations: Constructs and Measures. 2014:255.

21. Bolarinwa OA. Principles and methods of validity and reliability testing of questionnaires used in social and health science researches. The Nigerian postgraduate medical journal. 2015;22(4):195-201. Epub 2016/01/19. doi: https://doi.org/10.4103/1117-1936.173959. PubMed PMID: 26776330.

22. Fornell C, Larcker DF. Evaluating structural equation models with unobservable variables and measurement error. J Mark Res. 1981;18(1):39_ 50 .

23. Sharifi N, Khazaeian S, Pakzad R. Investigating the prevalence of preterm birth in Iranian population: a systematic review and meta-analysis. J Caring Sci. 2017;6(4):371.

24. Bottle A, Aylin P. Statistical methods for healthcare performance monitoring: CRC press; 2016

\section{Publisher's Note}

Springer Nature remains neutral with regard to jurisdictional claims in published maps and institutional affiliations.

Ready to submit your research? Choose BMC and benefit from:

- fast, convenient online submission

- thorough peer review by experienced researchers in your field

- rapid publication on acceptance

- support for research data, including large and complex data types

- gold Open Access which fosters wider collaboration and increased citations

- maximum visibility for your research: over $100 \mathrm{M}$ website views per year

At $\mathrm{BMC}$, research is always in progress.

Learn more biomedcentral.com/submissions 